\title{
Weight loss after Roux-En-Y gastric bypass surgery reveals skeletal muscle DNA methylation changes
}

Luis A. Garcia', Samantha E. Day², Richard L. Coletta ${ }^{1}$, Baltazar Campos', Tonya R. Benjamin³, Eleanna De Filippis ${ }^{3}$, James A. Madura $I^{4}$, Lawrence J. Mandarino ${ }^{1}$, Lori R. Roust ${ }^{3}$ and Dawn K. Coletta ${ }^{1,5^{*}}$ (D)

\begin{abstract}
Background: The mechanisms of weight loss and metabolic improvements following bariatric surgery in skeletal muscle are not well known; however, epigenetic modifications are likely to contribute. The aim of our study was to investigate skeletal muscle DNA methylation after weight loss induced by Roux-en-Y gastric bypass (RYGB) surgery. Muscle biopsies were obtained basally from seven insulin-resistant obese (BMI $>40 \mathrm{~kg} / \mathrm{m}^{2}$ ) female subjects (45.1 \pm 3.6 years) pre- and 3-month post-surgery with euglycemic hyperinsulinemic clamps to assess insulin sensitivity. Four lean $\left(\mathrm{BMl}<25 \mathrm{~kg} / \mathrm{m}^{2}\right.$ ) females (38.5 \pm 5.8 years) served as controls. We performed reduced representation bisulfite sequencing next generation methylation on DNA isolated from the vastus lateralis muscle biopsies.

Results: Global methylation was significantly higher in the pre- $(32.97 \pm 0.02 \%)$ and post-surgery $(33.31 \pm 0.02 \%)$ compared to the lean $(30.46 \pm 0.02 \%), P<0.05$. MethylSig analysis identified 117 differentially methylated cytosines (DMCs) that were significantly altered in the post- versus pre-surgery (Benjamini-Hochberg $q<0.05$ ). In addition, 2978 DMCs were significantly altered in the pre-surgery obese versus the lean controls (Benjamini-Hochberg $q<0.05$ ). For the post-surgery obese versus the lean controls, 2885 DMCs were altered (Benjamini-Hochberg $q<0.05$ ). Seven post-surgery obese DMCs were normalized to levels similar to those observed in lean controls. Of these, 5 were within intergenic regions (chr11.68,968,018, chr16.73,100,688, chr5.174,115,531, chr5.1,831,958 and chr9.98,547,011) and the remaining two DMCs chr17.45,330,989 and chr14.105,353,824 were within in the integrin beta 3 (ITGB3) promoter and KIAA0284 exon, respectively. ITGB3 methylation was significantly decreased in the post-surgery $(0.5 \pm 0.5 \%)$ and lean controls $(0 \pm 0 \%)$ versus pre-surgery $(13.6 \pm 2.7 \%, P<0.05)$. This decreased methylation post-surgery was associated with an increase in ITGB3 gene expression (fold change $+1.52, P=0.0087$ ). In addition, we showed that ITGB3 promoter methylation in vitro significantly suppressed transcriptional activity $(P<0.05)$. Transcription factor binding analysis for ITGB3 chr17.45,330,989 identified three putative transcription factor binding motifs; PAX-5, p53 and AP-2alphaA.

Conclusions: These results demonstrate that weight loss after RYGB alters the epigenome through DNA methylation. In particular, this study highlights ITGB3 as a novel gene that may contribute to the metabolic improvements observed post-surgery. Future additional studies are warranted to address the exact mechanism of ITGB3 in skeletal muscle.
\end{abstract}

\footnotetext{
*Correspondence: dcoletta@email.arizona.edu

1 Department of Medicine, Division of Endocrinology, The University

of Arizona College of Medicine, 1501 N. Campbell Ave, PO Box 245035,

Tucson, AZ 85724-5035, USA

Full list of author information is available at the end of the article
} original author(s) and the source, provide a link to the Creative Commons licence, and indicate if changes were made. The images or other third party material in this article are included in the article's Creative Commons licence, unless indicated otherwise in a credit line to the material. If material is not included in the article's Creative Commons licence and your intended use is not permitted by statutory regulation or exceeds the permitted use, you will need to obtain permission directly from the copyright holder. To view a copy of this licence, visit http://creativecommons.org/licenses/by/4.0/. The Creative Commons Public Domain Dedication waiver (http://creativeco mmons.org/publicdomain/zero/1.0/) applies to the data made available in this article, unless otherwise stated in a credit line to the data. 
Keywords: DNA methylation, Next generation sequencing, Skeletal muscle, Obesity, Bariatric surgery

\section{Background}

Obesity is a chronic and complex metabolic disease. Over the past 50 years, the prevalence of obesity has increased worldwide to epidemic proportions [1]. According to the World Health Organization (WHO), 39\% of adults were overweight in 2016, and $13 \%$ were obese. In the USA, the age-adjusted prevalence of obesity in adults was $42.4 \%$ [2]. Obesity is a major health challenge and increases the risk of a number of chronic diseases including type 2 diabetes, hypertension, dyslipidemia and cardiovascular disease [3]. People who are obese often present with hyperinsulinemia and an underlying insulin resistance, which increases their risk for type 2 diabetes [4]. For many patients, lifestyle modifications such as diet and exercise and pharmacological therapies are ineffective at achieving long-term weight loss. Another alternative for obesity treatment is bariatric surgery. Bariatric surgery is a weight loss treatment for patients with a BMI of greater than or equal to $35 \mathrm{~kg} / \mathrm{m}^{2}$ along with underlying comorbidities or with a body mass index (BMI) greater than or equal to $40 \mathrm{~kg} / \mathrm{m}^{2}$. We [5] and others [6-13] showed that bariatric surgery results in weight loss along with metabolic improvements. The metabolic benefits of surgical intervention include improved blood glucose levels and improvements in insulin sensitivity and secretion [5, 1113]. In addition, a meta-analysis across 621 studies with 135,246 patients revealed that type 2 diabetes is resolved in the greater majority of patients following bariatric surgery [14].

The molecular mechanism by which insulin exerts its actions in skeletal muscle has led to a greater and detailed understanding of insulin signaling and glucose transport [15-19]. However, the mechanisms to explain the metabolic improvements following weight loss induced by bariatric surgery in skeletal muscle are less known. We previously showed a global analysis of protein abundance changes in combination with transcriptomic analyses in skeletal muscle following Rouxen-Y gastric bypass (RYGB) surgery [5]. In that study, we showed that bariatric surgery results in skeletal muscle changes for genes and proteins involved in insulin signaling and ribosomal pathways [5]. We also showed that the cytoskeletal interacting protein sorbin and $\mathrm{SH} 3$ domain containing 3 (SORBS3) was decreased in methylation in the skeletal muscle of patients following weight loss induced by RYGB [20]. Gastaldi et al. showed that weight loss induced by RYGB resulted in an upregulation of the peroxisome proliferator-activated receptor gamma coactivator (PPARGC1A) gene [21]. In another study, it was demonstrated that insulin signaling genes were increased in skeletal muscle following bariatric surgery [22]. Barres et al. showed that obesity was associated with altered expression of a number of genes coding for metabolic and mitochondrial proteins [11]. In that same study, they showed that surgery-induced weight loss resulted in a normalization of those genes to levels observed in healthy, weight controls [11].

The effects of bariatric surgery on the epigenome of skeletal muscle is less known. To our knowledge, there has been only one study that investigated global methylation changes in patients before and after bariatric surgery. The study of Barres et al. showed that global methylation in human skeletal muscle is unaltered by either obesity or RYGB surgery-induced weight loss [11]. However, in that same study, the authors performed promoter-specific DNA methylation of a subset of metabolic genes. Among the 14 metabolic genes analyzed, promoter methylation of 11 genes including PPARGC1A and pyruvate dehydrogenase kinase 4 (PDK4) were normalized to levels observed in the healthy weight controls [11]. The aim of the present study was to determine the global DNA methylation changes that occurred in skeletal muscle following RYGB. RYGB is one of the most common surgeries performed to treat obesity and combines restrictive and malabsorptive techniques. The focus of this study was on the epigenetic marker DNA methylation, because the addition or removal of the methyl marks may influence and regulate gene expression. For this study, we hypothesized that bariatric surgery would alter the skeletal muscle epigenome of genes involved in insulin signaling and ribosomal pathways, in part because our previous transcriptomic and proteomic analyses revealed these changes following bariatric surgery [5]. Our global methylation analysis approach allowed us to test this specific hypothesis while at the same time performing a genomewide approach that revealed novel and unanticipated findings.

\section{Results \\ Participants}

Table 1 shows the anthropometric and clinical characteristics for the RYGB participants post- versus pre-surgery and the lean healthy controls. The metabolic data for the RYGB participants post- versus pre-surgery have been described in a previous publication [5]. Briefly, 3-month post-surgery, significant improvements were observed in BMI, body fat percentage, cholesterol, low-density lipoprotein (LDL), fasting plasma glucose (FPG) and fasting 
Table 1 Participant characteristics

\begin{tabular}{|c|c|c|c|c|c|c|}
\hline & Lean & Pre-surgery obese & Post-surgery obese & $\begin{array}{l}P \text { value } \\
\text { versus Lean } \\
\text { Pre }\end{array}$ & $\begin{array}{l}P \text { value } \\
\text { Pre versus Post }\end{array}$ & $\begin{array}{l}P \text { value } \\
\text { Lean versus Post }\end{array}$ \\
\hline Sex & Four female & Seven female & & & & \\
\hline Age (years) & $38.5 \pm 5.8$ & $45.1 \pm 3.6$ & $45.3 \pm 3.5$ & NS & NS & NS \\
\hline Body mass index $\left(\mathrm{kg} / \mathrm{m}^{2}\right)$ & $23.2 \pm 0.9$ & $42.1 \pm 2.2$ & $35.3 \pm 1.8$ & $<0.001$ & $<0.001$ & $<0.01$ \\
\hline Body fat (\%) & $30.3 \pm 1.0$ & $46.4 \pm 1.2$ & $40.6 \pm 1.3$ & $<0.00001$ & $<0.01$ & $<0.001$ \\
\hline Waist circumference (cm) & $83.5 \pm 3.2$ & $122.7 \pm 6.3$ & $109.4 \pm 109.4$ & $<0.01$ & $<0.0001$ & $<0.05$ \\
\hline Systolic blood pressure (mmHg) & $102.3 \pm 12.6$ & $125.1 \pm 3.9$ & $119.1 \pm 4.6$ & NS & NS & NS \\
\hline Diastolic blood pressure $(\mathrm{mmHg})$ & $59.8 \pm 13.3$ & $71.7 \pm 2.0$ & $75.1 \pm 1.7$ & NS & NS & NS \\
\hline Triglycerides (mg/dL) & $92.8 \pm 7.8$ & $121.9 \pm 17.5$ & $107.7 \pm 11.2$ & NS & NS & NS \\
\hline Cholesterol (mg/dL) & $202.3 \pm 11.4$ & $181.4 \pm 13.2$ & $151.5 \pm 11.2$ & NS & $<0.01$ & $<0.05$ \\
\hline High-density lipoprotein (mg/dL) & $63.0 \pm 9.0$ & $45.0 \pm 2.7$ & $45.0 \pm 2.5$ & $<0.05$ & NS & $<0.05$ \\
\hline Low-density lipoprotein (mg/dL) & $120.8 \pm 7.6$ & $112.1 \pm 11.9$ & $84.8 \pm 10.5$ & NS & $<0.01$ & $<0.05$ \\
\hline Hemoglobin A1c \% & $5.3 \pm 0.1$ & $6.0 \pm 0.2$ & $5.7 \pm 0.1$ & $<0.05$ & NS & $<0.05$ \\
\hline Fasting plasma glucose (mg/dL) & $87.2 \pm 3.1$ & $104.2 \pm 7.8$ & $86.7 \pm 3.1$ & NS & $<0.05$ & NS \\
\hline Fasting serum insulin (ulU/mL) & $7.9 \pm 4.2$ & $18.2 \pm 2.7$ & $7.5 \pm 1.0$ & $<0.01$ & $<0.01$ & NS \\
\hline M-value (mg/kg/min) & $6.7 \pm 0.7$ & $2.4 \pm 0.3$ & $2.9 \pm 0.4$ & $<0.001$ & NS & $<0.001$ \\
\hline
\end{tabular}

Data are mean \pm SEM

serum insulin (FSI) (Table 1). There were no significant improvements in blood pressure, triglycerides, highdensity lipoprotein (HDL), hemoglobin A1c (HbA1c) and insulin-stimulated glucose disposal (M-value) following RYGB surgery (Table 1). As expected, the lean controls had significantly lower BMI, body fat percentage and HbA1c compared to the pre-surgery participants. The lean controls showed significantly higher levels of insulin-stimulated glucose disposal and HDL compared to the pre-surgery participants. The FSI post-surgery was normalized to levels similar to those observed in lean controls (Table 1).

\section{Methylation sites distribution}

Prior to the quality control of the sequence data, 5,003,481 sites were captured across the MethylSig data object for the lean, pre-surgery obese and post-surgery obese RRBS datasets. A threshold of greater than $80 \%$ call rate and a minimum of $5 \mathrm{X}$ coverage for the RRBS data was set. In total, $2,821,706 \pm 116,015$ methylation sites were captured across the methylation datasets when this threshold was set. The distribution of the methylation sites across the three datasets was defined by genic regions (Fig. 1a) and CpG Island features (Fig. 1b). The majority of the methylation sites were in intergenic (29\%), intronic (24\%) and promoter (19\%) regions (Fig. 1a). Sites in the promoter and $5^{\prime}$ untranslated regions (UTR) dominantly overlapped with CpG islands (Fig. 1b). As shown in Fig. 2, global methylation was significantly higher postversus pre-surgery $(33.31 \pm 0.02 \%$ versus $32.97 \pm 0.02 \%$,
$P<0.05)$. In addition, global methylation was significantly lower in the lean participants $(30.46 \pm 0.02 \%)$ compared with post- and pre-surgery $(P<0.05)$. A summary of the methylation analysis findings is shown in Fig. 3.

\section{Post- versus pre-surgery methylation analyses}

MethylSig analysis revealed 117 differentially methylated cytosines (DMCs) that were significantly altered in methylation post-surgery versus pre-surgery (BenjaminiHochberg $q<0.05)$ Of the 117 DMCs, 75 were decreased and 42 were increased post-surgery. Of the decreasers and increasers, 45 and 28 were assigned to a gene, respectively. The remaining DMCs were not associated with a gene. Table 2 shows the 45 genes with significant (Benjamini-Hochberg $q<0.05)$ DMCs that were decreased post-versus pre-surgery. Table 3 shows the 28 genes with significant (Benjamini-Hochberg $q<0.05$ ) DMCs that were increased post-surgery versus pre-surgery.

There were no KEGG pathways enriched in either the decreased or increased DMC genes in the post-versus pre-surgery analyses. DAVID gene ontology analysis of the DMC genes that was decreased in the post-surgery group revealed an enrichment in 11 functional categories (Additional file 1). The top two significant functional groups were DNA binding (TNFRSF6B, ZNF275, NDN, PRR12, TBX1, POLRMT, ISL2, HOXC12, HOXA3, HOXA4, ZNF317, SIM1 and PITX2) and regulation of RNA metabolic process (ZNF275, ISL2, HOXC12, HOXA3, HOXA4, NDN, ZNF317, CASK, TBX1, PABPC1, SIM1 and PITX2). Moreover, Additional file 1 


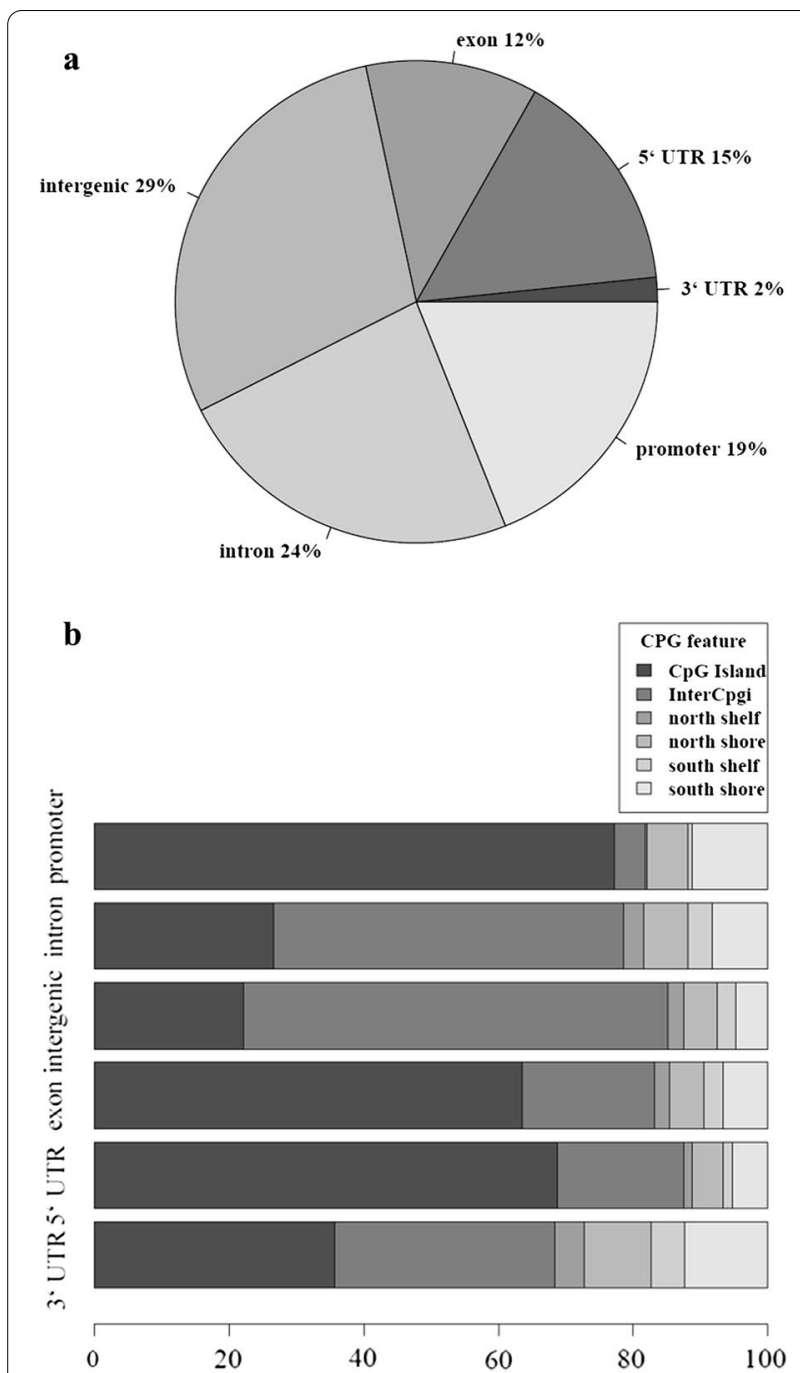

Fig. 1 Methylation sites captured were mapped in the context of gene regions (a) and $\mathrm{CpG}$ island features $(\mathbf{b})$. The regions were defined using UCSC browser refGene and CpG island tracks. The promoter region was defined as $1000 \mathrm{bp}$ (basepairs) upstream of the transcription start site (TSS); untranslated region (UTR); CPG island is 200-3000 bp stretch of DNA with a C + G content of $50 \%$ and observed CpG/expected CpG in excess of 0.6; North (N) and South (S) shores flank the CpG island by 0-2000 bp; the North (N) and South (S) shelf flank the shores by $2000 \mathrm{bp}$ (2000-4000 bp from the island)

shows a number of developmental functional groups identified including skeletal system morphogenesis and embryonic skeletal system development/morphogenesis. DAVID gene ontology analysis on the genes with increased DMCs post-surgery identified 11 enriched groups (Additional file 2). The heat shock protein binding (FAF1, DNAJC5G and DNAJC3) was the most significantly enriched group. Interestingly, the increased DMC genes OBSCN, AR, FAF1, TRAF6 and NLRP1

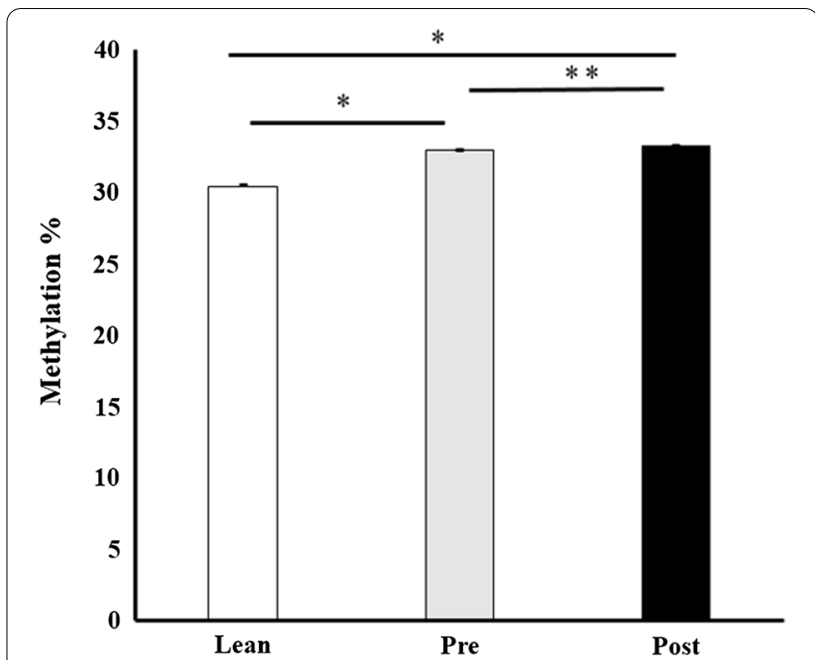

Fig. 2 Reduced representation bisulfite sequencing analysis of global DNA methylation of DNA extracted from muscle in lean participants and patients pre- versus post-surgery. Data are mean \pm SEM. ${ }^{*} P<0.05$ using Mann-Whitney $U$ test. ${ }^{*} P<0.05$ using Wilcoxon signed-rank test

grouped together in functional groups related to positive regulation of cell death and positive regulation of apoptosis (Additional file 2).

\section{Pre-surgery obese versus lean methylation analyses}

MethylSig analysis revealed 2,978 differentially methylated sites (DMCs) that were significantly altered in the pre-surgery obese versus the lean controls (Benjamini-Hochberg $q<0.05$ ) (Additional file 3). Of the 2,978 DMCs, 1,519 were decreased in methylation in the presurgery obese group compared to the lean controls (1047 DMCs were assigned to a gene, Additional file 3). In addition, 1,459 DMCs were increased in methylation in the pre-surgery obese versus the lean group (944 DMCs were assigned to a gene, Additional file 3).

KEGG pathway analysis of the DMC genes that were decreased in the pre-surgery obese group revealed an enrichment in 11 pathways (Additional file 4), including regulation of actin cytoskeleton (hsa04810), focal adhesion (hsa04510), insulin signaling pathway (hsa04910) and adipocytokine signaling pathway (hsa04920). There were 6 KEGG pathways enriched in the increased DMC genes in the pre-surgery obese group including longterm potentiation (hsa04720), phosphatidylinositol signaling system (hsa04070), chemokine signaling pathway (hsa04062) and calcium signaling pathway (hsa04020). Interestingly, the focal adhesion pathway (hsa04510) was also enriched in the increased DMC genes (Additional file 5). 


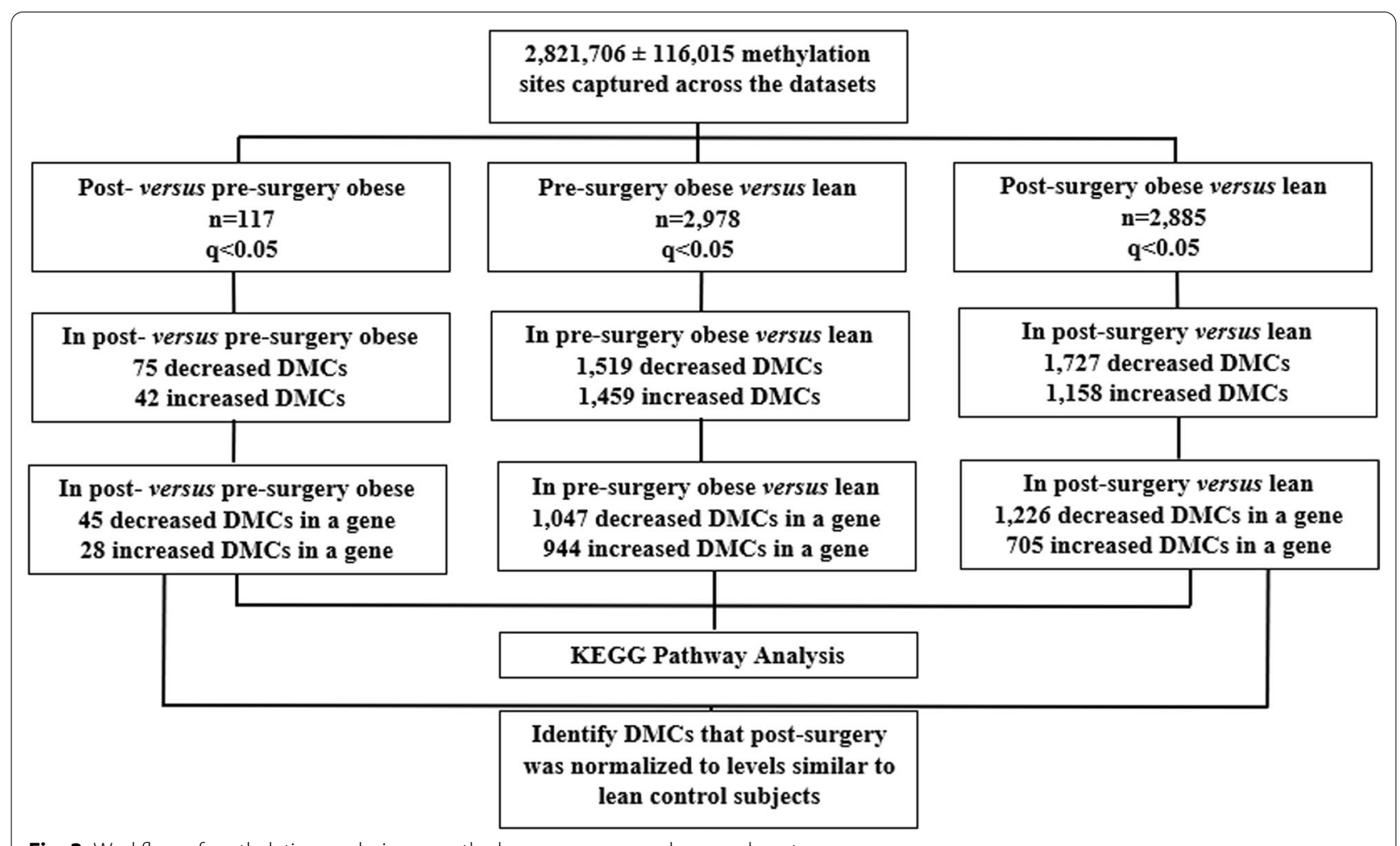

Fig. 3 Workflow of methylation analysis across the lean, pre-surgery obese and post-surgery groups

Post-surgery obese versus lean methylation analyses

MethylSig analysis revealed 2,885 differentially methylated sites (DMCs) that were significantly altered in the post-surgery obese versus the lean controls (Benjamini-Hochberg $q<0.05$ ) (Additional file 6). Of the 2,885 DMCs, 1,727 were decreased in methylation in the postsurgery obese group compared to the lean controls $(1,226$ DMCs were assigned to a gene, Additional file 6). In addition, 1,158 DMCs were increased in methylation in the post-surgery obese versus the lean group (705 DMCs were assigned to a gene, Additional file 6).

KEGG pathway analysis of the DMC genes that were decreased in the post-surgery obese group revealed an enrichment in 24 pathways (Additional file 7), including regulation of actin cytoskeleton (hsa04810), focal adhesion (hsa04510), ECM-receptor interaction (hsa04512), PI3K-Akt signaling pathway (hsa04151), insulin resistance (hsa04931), adipocytokine signaling pathway (hsa04920), MAPK signaling pathway (hsa04010), insulin secretion (hsa04911), insulin signaling pathway (hsa04910) and AMPK signaling pathway (hsa04152). There were 6 KEGG pathways enriched in the increased DMC genes in the post-surgery obese group including calcium signaling pathway (hsa04020), neuroactive ligand-receptor interaction (hsa04080), notch signaling pathway (hsa04330) and mucin type O-glycan biosynthesis (hsa00512) (Additional file 8).

Post-surgery DMCs normalized to lean control methylation analyses

We identified 7 DMCs that post-surgery were normalized to levels similar to those observed in the lean control subjects (Fig. 4). In order to be considered normalized, we stipulated that DMCs had to be significantly (BenjaminiHochberg $q<0.05$ ) different in both the pre-surgery obese versus lean and post- versus pre-surgery analyses. Of the 7 DMCs, five were in intergenic regions at positions chr11.68,968,018, chr16.73,100,688, chr5.174,115,531, chr5.1,831,958 and chr9.98,547,011. The remaining two DMCs chr17.45,330,989 and chr14.105,353,824 were associated with ITGB3 and KIAA0284 gene, respectively. The ITGB3 DMC was within a promoter region and KIAA0284 DMC was in an exonic region.

\section{ITGB3 gene expression in post- versus pre-surgery}

The quantitative RT-PCR results showed an increase in gene expression of ITGB3 post-surgery compared to pre-surgery $(+1.52$ versus 1.0 -fold change, respectively; $P=0.0087)$. Skeletal muscle RNA was not 
Table 2 Differentially methylated cytosines (DMCs; $q<0.05)$ decreased post-surgery versus pre-surgery

\begin{tabular}{|c|c|c|c|c|c|c|c|c|}
\hline Chr & Position & Gene & $\begin{array}{l}\text { Pre-surgery } \\
\text { methylation \% }\end{array}$ & $\begin{array}{l}\text { Post-surgery } \\
\text { methylation \% }\end{array}$ & $P$ value & $q$ value & Genic region & CpG region \\
\hline chr19 & $9,271,227$ & ZNF317 & $99.54 \pm 0.46$ & $88.87 \pm 2.27$ & 8.86E-09 & 0.0020 & Exon & CpG_Island \\
\hline $\operatorname{chrX}$ & $41,782,532$ & CASK & $36.44 \pm 4.47$ & $7.49 \pm 1.49$ & $1.61 \mathrm{E}-08$ & 0.0028 & Promoter & CpG_Island \\
\hline chr9 & $134,346,329$ & PRRC2B & $99.15 \pm 0.57$ & $86.92 \pm 2.80$ & $1.69 E-08$ & 0.0028 & Exon & InterCpgi \\
\hline chr15 & $101,420,903$ & ALDH1A3 & $81.98 \pm 2.87$ & $58.77 \pm 5.28$ & $3.90 \mathrm{E}-08$ & 0.0050 & Intron & CpG_Island \\
\hline chr3 & $13,696,951$ & LOC285375 & $9.02 \pm 2.58$ & $0 \pm 0$ & $3.63 \mathrm{E}-08$ & 0.0050 & Intergenic & CpG_Island \\
\hline chr11 & $70,008,217$ & ANO1 & $99.47 \pm 0.53$ & $82.21 \pm 2.68$ & $6.57 \mathrm{E}-08$ & 0.0079 & Intron & InterCpgi \\
\hline chr5 & $9,509,512$ & SEMA5A & $100 \pm 0$ & $91.29 \pm 2.45$ & $8.58 \mathrm{E}-08$ & 0.0091 & utr5 & InterCpgi \\
\hline chr19 & $48,824,452 \neq$ & CCDC114 & $79.96 \pm 2.47$ & $52.67 \pm 3.92$ & $1.11 \mathrm{E}-07$ & 0.0105 & Promoter & North_shore \\
\hline chr19 & $48,824,452 \neq$ & EMP3 & $79.96 \pm 2.47$ & $52.67 \pm 3.92$ & $1.11 \mathrm{E}-07$ & 0.0105 & Promoter & North_shore \\
\hline chr15 & $76,634,525$ & ISL2 & $6.79 \pm 1.30$ & $0 \pm 0$ & $1.09 \mathrm{E}-07$ & 0.0105 & utr3 & CpG_Island \\
\hline chr16 & $1,774,262$ & MAPK8IP3 & $96.07 \pm 0.89$ & $86.94 \pm 2.61$ & $1.36 \mathrm{E}-07$ & 0.0116 & Intron & InterCpgi \\
\hline chr15 & $62,457,572$ & $\mathrm{C} 2 \mathrm{CD} 4 \mathrm{~B}$ & $52.02 \pm 4.77$ & $15.90 \pm 4.03$ & $1.48 \mathrm{E}-07$ & 0.0122 & Promoter & CpG_Island \\
\hline chr16 & $89,687,436$ & DPEP1 & $74.63 \pm 2.21$ & $46.44 \pm 4.95$ & $1.80 E-07$ & 0.0135 & utr5 & InterCpgi \\
\hline chr17 & $45,330,989$ & ITGB3 & $13.64 \pm 2.74$ & $0.51 \pm 0.51$ & $1.96 \mathrm{E}-07$ & 0.0135 & Promoter & CpG_Island \\
\hline chr9 & $104,075,142$ & LPPR1 & $100 \pm 0$ & $82.89 \pm 5.22$ & $2.13 \mathrm{E}-07$ & 0.0135 & Exon & InterCpgi \\
\hline chr16 & $30,990,627$ & SETD1A & $86.89 \pm 3.56$ & $57.56 \pm 5.29$ & $1.84 \mathrm{E}-07$ & 0.0135 & Exon & North_shore \\
\hline chr12 & $54,345,965$ & $\mathrm{HOXC12}$ & $15.68 \pm 1.11$ & $3.11 \pm 1.14$ & $2.69 \mathrm{E}-07$ & 0.0159 & Intergenic & South_shore \\
\hline chr19 & 619,189 & POLRMT & $99.40 \pm 0.6$ & $91.17 \pm 1.00$ & $3.34 \mathrm{E}-07$ & 0.0188 & Intron & CpG_Island \\
\hline chr11 & $628,172^{\ddagger}$ & CDHR5 & $100 \pm 0$ & $89.48 \pm 2.13$ & $3.74 \mathrm{E}-07$ & 0.0192 & Promoter & South_shore \\
\hline chr11 & $628,172^{\ddagger}$ & $\mathrm{SCT}$ & $100 \pm 0$ & $89.48 \pm 2.13$ & $3.74 \mathrm{E}-07$ & 0.0192 & Promoter & South_shore \\
\hline chr10 & $88,269,670$ & WAPAL & $99.68 \pm 0.32$ & $91.58 \pm 1.90$ & $3.76 \mathrm{E}-07$ & 0.0192 & Intron & InterCpgi \\
\hline chr1 & $235,979,239$ & LYST & $42.96 \pm 5.36$ & $15.09 \pm 3.25$ & $3.94 \mathrm{E}-07$ & 0.0197 & Intron & InterCpgi \\
\hline chr19 & $50,098,039$ & PRR12 & $87.92 \pm 1.83$ & $67.43 \pm 3.66$ & $4.39 E-07$ & 0.0216 & Exon & CpG_Island \\
\hline chr8 & $101,723,031$ & PABPC1 & $83.96 \pm 3.70$ & $42.12 \pm 5.68$ & $4.62 \mathrm{E}-07$ & 0.0219 & Intron & InterCpgi \\
\hline chr15 & $23,932,055$ & NDN & $39.77 \pm 5.54$ & $14.89 \pm 3.47$ & $4.81 \mathrm{E}-07$ & 0.0222 & Exon & CpG_Island \\
\hline chr5 & $1,491,652$ & LPCAT1 & $98.22 \pm 1.19$ & $79.85 \pm 3.10$ & $5.04 \mathrm{E}-07$ & 0.0226 & Intron & South_shore \\
\hline chr11 & $61,486,429$ & DAGLA & $58.08 \pm 3.83$ & $34.62 \pm 3.51$ & $5.53 \mathrm{E}-07$ & 0.0241 & utr5 & North_shore \\
\hline chr3 & $126,746,778$ & PLXNA1 & $100 \pm 0$ & $87.57 \pm 1.95$ & 5.87E-07 & 0.0251 & Intron & North_shore \\
\hline chr15 & $23,892,609$ & MAGEL2 & $54.77 \pm 8.17$ & $19.70 \pm 3.99$ & $6.66 \mathrm{E}-07$ & 0.0276 & Exon & InterCpgi \\
\hline chr4 & $111,553,834$ & PITX2 & $88.67 \pm 3.28$ & $55.56 \pm 9.64$ & 7.47E-07 & 0.0297 & Intron & CpG_Island \\
\hline chr17 & $79,497,291$ & FSCN2 & $57.24 \pm 5.14$ & $29.65 \pm 3.61$ & $8.94 \mathrm{E}-07$ & 0.0308 & Intron & South_shore \\
\hline chr14 & $24,666,733$ & TM9SF1 & $93.83 \pm 2.06$ & $71.93 \pm 3.85$ & $8.75 \mathrm{E}-07$ & 0.0308 & Promoter & South_shore \\
\hline chr21 & $43,859,102$ & UBASH3A & $86.97 \pm 2.24$ & $68.44 \pm 4.45$ & $8.62 \mathrm{E}-07$ & 0.0308 & Intron & InterCpgi \\
\hline $\operatorname{chr} x$ & $152,600,028$ & ZNF275 & $48.08 \pm 2.35$ & $25.34 \pm 3.90$ & $8.31 E-07$ & 0.0308 & utr5 & CpG_Island \\
\hline chr4 & $3,678,810$ & LOC 100133461 & $93.87 \pm 2.40$ & $63.82 \pm 6.14$ & $9.43 \mathrm{E}-07$ & 0.0317 & Intergenic & InterCpgi \\
\hline chr13 & $67,135,465$ & PCDH9 & $98.97 \pm 0.50$ & $89.39 \pm 1.64$ & $1.14 \mathrm{E}-06$ & 0.0370 & Intron & InterCpgi \\
\hline $\operatorname{chr} x$ & $10,188,915$ & CLCN4 & $100 \pm 0$ & $91.75 \pm 2.13$ & $1.42 \mathrm{E}-06$ & 0.0419 & Exon & InterCpgi \\
\hline chr18 & $34,274,365$ & FHOD3 & $59.66 \pm 4.19$ & $34.53 \pm 3.20$ & $1.51 \mathrm{E}-06$ & 0.0429 & Intron & InterCpgi \\
\hline chr20 & $62,329,849$ & TNFRSF6B & $76.74 \pm 2.63$ & $57.78 \pm 4.65$ & $1.62 E-06$ & 0.0448 & Exon & South_shore \\
\hline chr15 & $101,421,027$ & ALDH1A3 & $79.81 \pm 3.85$ & $54.34 \pm 5.33$ & $2.04 \mathrm{E}-06$ & 0.0479 & Intron & CpG_Island \\
\hline chr20 & $3,778,167$ & CDC25B & $95.43 \pm 1.99$ & $75.05 \pm 3.37$ & $1.90 \mathrm{E}-06$ & 0.0479 & utr5 & South_shore \\
\hline chr7 & $27169037^{\wedge}$ & HOXA3 & $94.58 \pm 1.54$ & $78.97 \pm 4.24$ & $1.96 \mathrm{E}-06$ & 0.0479 & Exon & North_shore \\
\hline chr7 & $27169037^{\wedge}$ & HOXA4 & $94.58 \pm 1.54$ & $78.97 \pm 4.24$ & $1.96 \mathrm{E}-06$ & 0.0479 & Exon & north_shore \\
\hline chr6 & $100,906,424$ & SIM1 & $49.04 \pm 2.93$ & $18.82 \pm 4.42$ & $2.00 \mathrm{E}-06$ & 0.0479 & Intron & CpG_Island \\
\hline chr22 & $19,747,159$ & $\mathrm{TBX} 1$ & $44.30 \pm 4.95$ & $22.56 \pm 2.96$ & $2.14 \mathrm{E}-06$ & 0.0498 & utr5 & South_shore \\
\hline
\end{tabular}

Data are mean \pm SEM. The $P$ value is calculated from the MethylSig analysis. The $q$ value is generated by Benjamini-Hochberg multiple testing correction

${ }^{\ddagger}$ Two genes [SCT and CDHR5] are associated with the same differentially methylated cytosine [Chr11:628172]; $\neq$ Two genes [EMP3 and CCDC114] are associated with the same differentially methylated cytosine [Chr19: 48824452]

* Two genes [EMP3 and CCDC114] are associated with the same differentially methylated cytosine [Chr 19: 48824452]

${ }^{\wedge}$ Two genes [HOXA3 and HOXA4] are associated with the same differentially methylated cytosine [Chr7: 27169037] 
Table 3 Differentially methylated cytosines (DMCs; $q<0.05)$ that were increased post-surgery versus pre-surgery

\begin{tabular}{|c|c|c|c|c|c|c|c|c|}
\hline Chr & Position & Gene & $\begin{array}{l}\text { Pre-surgery } \\
\text { methylation \% }\end{array}$ & $\begin{array}{l}\text { Post-surgery } \\
\text { methylation \% }\end{array}$ & $P$ value & $q$ value & Genic region & CpG region \\
\hline chr2 & $27,498,318$ & DNAJC5G & $51.83 \pm 9.21$ & $89.02 \pm 2.98$ & $1.01 \mathrm{E}-09$ & 0.00067 & utr5 & InterCpgi \\
\hline chr7 & $20,830,624$ & SP8 & $1.30 \pm 0.84$ & $13.90 \pm 1.02$ & $2.01 \mathrm{E}-09$ & 0.00076 & Intergenic & CpG_Island \\
\hline chr1 & $228,504,058$ & OBSCN & $42.29 \pm 6.19$ & $84.61 \pm 2.99$ & $3.28 \mathrm{E}-09$ & 0.00109 & Intron & CpG_Island \\
\hline chr10 & $119,271,421$ & EMX2OS & $42.57 \pm 2.47$ & $68.77 \pm 3.23$ & $6.08 \mathrm{E}-09$ & 0.00161 & Intergenic & InterCpgi \\
\hline chr1 & $51,154,377$ & FAF1 & $92.12 \pm 2.38$ & $100 \pm 0$ & $1.16 \mathrm{E}-08$ & 0.00220 & Intron & InterCpgi \\
\hline chr2 & $11,925,218$ & LPIN1 & $0 \pm 0$ & $9.68 \pm 1.23$ & $1.55 \mathrm{E}-07$ & 0.01243 & Intron & CpG_Island \\
\hline chr1 & $240,347,650$ & FMN2 & $84.75 \pm 4.18$ & $99.35 \pm 0.65$ & $2.06 \mathrm{E}-07$ & 0.01352 & Intron & InterCpgi \\
\hline chr4 & $57,068,530$ & KIAA1211 & $83.59 \pm 2.29$ & $98.25 \pm 1.13$ & $1.89 \mathrm{E}-07$ & 0.01352 & utr5 & InterCpgi \\
\hline chr18 & $13,431,993$ & C18orf1 & $84.39 \pm 3.53$ & $100 \pm 0$ & $3.16 \mathrm{E}-07$ & 0.01820 & Intron & InterCpgi \\
\hline chr3 & $113,160,431$ & WDR52 & $13.71 \pm 2.73$ & $46.89 \pm 6.19$ & $3.49 \mathrm{E}-07$ & 0.01886 & Promoter & CpG_Island \\
\hline chr13 & $96,414,410$ & DNAJC3 & $90.20 \pm 2.64$ & $100 \pm 0$ & $5.33 \mathrm{E}-07$ & 0.02357 & Intron & InterCpgi \\
\hline chr1 & $197,108,554$ & ASPM & $86.50 \pm 4.35$ & $100 \pm 0$ & $7.84 \mathrm{E}-07$ & 0.03038 & Intron & InterCpgi \\
\hline chr17 & $5,462,805$ & NLRP1 & $80.80 \pm 5.66$ & $99.05 \pm 0.95$ & $7.90 \mathrm{E}-07$ & 0.03038 & Exon & InterCpgi \\
\hline chr19 & $54,872,598$ & LAIR1 & $67.12 \pm 6.27$ & $93.87 \pm 2.11$ & $8.60 \mathrm{E}-07$ & 0.03079 & Exon & InterCpgi \\
\hline chr11 & $36,512,043$ & TRAF6 & $40.53 \pm 4.68$ & $65.66 \pm 3.25$ & $8.80 \mathrm{E}-07$ & 0.03079 & Exon & InterCpgi \\
\hline chr15 & $52,248,473$ & LEO1 & $18.60 \pm 3.51$ & $45.53 \pm 4.24$ & $9.21 \mathrm{E}-07$ & 0.03131 & Intron & InterCpgi \\
\hline chr22 & $50,919,564$ & ADM2 & $2.23 \pm 0.81$ & $11.87 \pm 1.89$ & $1.17 \mathrm{E}-06$ & 0.03742 & Promoter & CpG_Island \\
\hline chr7 & $135,258,846$ & NUP205 & $88.18 \pm 3.66$ & $100 \pm 0$ & $1.20 E-06$ & 0.03793 & Intron & InterCpgi \\
\hline chr1 & $93,172,465$ & EVI5 & $83.13 \pm 3.46$ & $100 \pm 0$ & $1.42 \mathrm{E}-06$ & 0.04189 & Intron & InterCpgi \\
\hline chr12 & $133,125,172$ & FBRSL1 & $88.19 \pm 3.33$ & $100 \pm 0$ & $1.44 \mathrm{E}-06$ & 0.04203 & intron & North_shore \\
\hline chr5 & $137,729,043$ & KDM3B & $84.36 \pm 3.76$ & $96.62 \pm 1.45$ & $1.52 \mathrm{E}-06$ & 0.04294 & Exon & InterCpgi \\
\hline chr3 & $9,989,586$ & PRRT3 & $45.47 \pm 3.64$ & $74.18 \pm 3.58$ & $1.49 \mathrm{E}-06$ & 0.04294 & Exon & CpG_Island \\
\hline $\operatorname{chr} x$ & $66,764,054$ & $\mathrm{AR}$ & $15.74 \pm 4.49$ & $42.72 \pm 2.58$ & $1.67 \mathrm{E}-06$ & 0.04508 & utr5 & CpG_Island \\
\hline chr1 & $91,184,127$ & BARHL2 & $0 \pm 0$ & $2.97 \pm 0.83$ & $1.81 \mathrm{E}-06$ & 0.04740 & Promoter & CpG_Island \\
\hline chr7 & 939,123 & ADAP1 & $40.13 \pm 9.99$ & $74.26 \pm 3.10$ & $1.83 \mathrm{E}-06$ & 0.04759 & Exon & South_shelf \\
\hline chr1 & $34,642,648$ & C1orf94 & $0 \pm 0$ & $7.35 \pm 2.25$ & $2.04 \mathrm{E}-06$ & 0.04785 & utr5 & CpG_Island \\
\hline chr7 & $71,562,951$ & CALN1 & $81.90 \pm 4.23$ & $98.70 \pm 1.30$ & $1.96 \mathrm{E}-06$ & 0.04785 & Intron & InterCpgi \\
\hline chr14 & $105,353,824$ & KIAA0284 & $62.07 \pm 7.36$ & $90.15 \pm 4.41$ & $1.93 \mathrm{E}-06$ & 0.04785 & Exon & South_shelf \\
\hline
\end{tabular}

Data are mean \pm SEM. The $P$ value is calculated from the MethylSig analysis. The $q$ value is generated by Benjamini-Hochberg multiple testing correction

available to perform the gene expression analysis on the lean participants.

\section{ITGB3 promoter methylation in vitro alters reporter gene expression}

The ITGB3 construct was created to test the effect of DNA methylation on transcriptional activity. The level of suppressed transcriptional activity, as measured by luciferase activity, was determined in comparison to the mock methylated control (Fig. 5). As shown in Fig. 5, when the ITGB3 construct was methylated in vitro using the HhaI enzyme (GCGC, $n=6$ sites), transcriptional activity was suppressed by $52.8 \%$. Moreover, when the ITGB3 construct was methylated in vitro using the SssI enzyme (CG, $n=53$ sites), transcriptional activity was suppressed by $73.4 \%$. There was a statistically significant difference between the groups as determined by a Kruskal-Wallis test (Chi square $=20.931, P=0.0000285, \mathrm{df}=2$ ).

\section{Predicted transcription factor binding analysis for ITGB3} chr17.45,330,989

PROMO analysis identified three potential transcription factor binding motifs overlapping the differentially methylated cytosine (chr17.45,330,989) of ITGB3. The transcription factors were PAX-5, p53 and AP-2alphaA.

\section{Discussion}

The overarching goal of the present study was to determine whether global DNA methylation changes occur in skeletal muscle following Roux-en-Y gastric bypass (RYGB). We previously published the metabolic changes observed in these patients post- versus pre-surgery [5]; therefore, the focus of this paper was on the epigenetic changes that occur with bariatric surgery. As part of this study, we were able to capture overall global methylation post- versus pre-surgery and also identify novel differentially methylated cytosines. We included a lean control group, in part to identify differentially methylated 


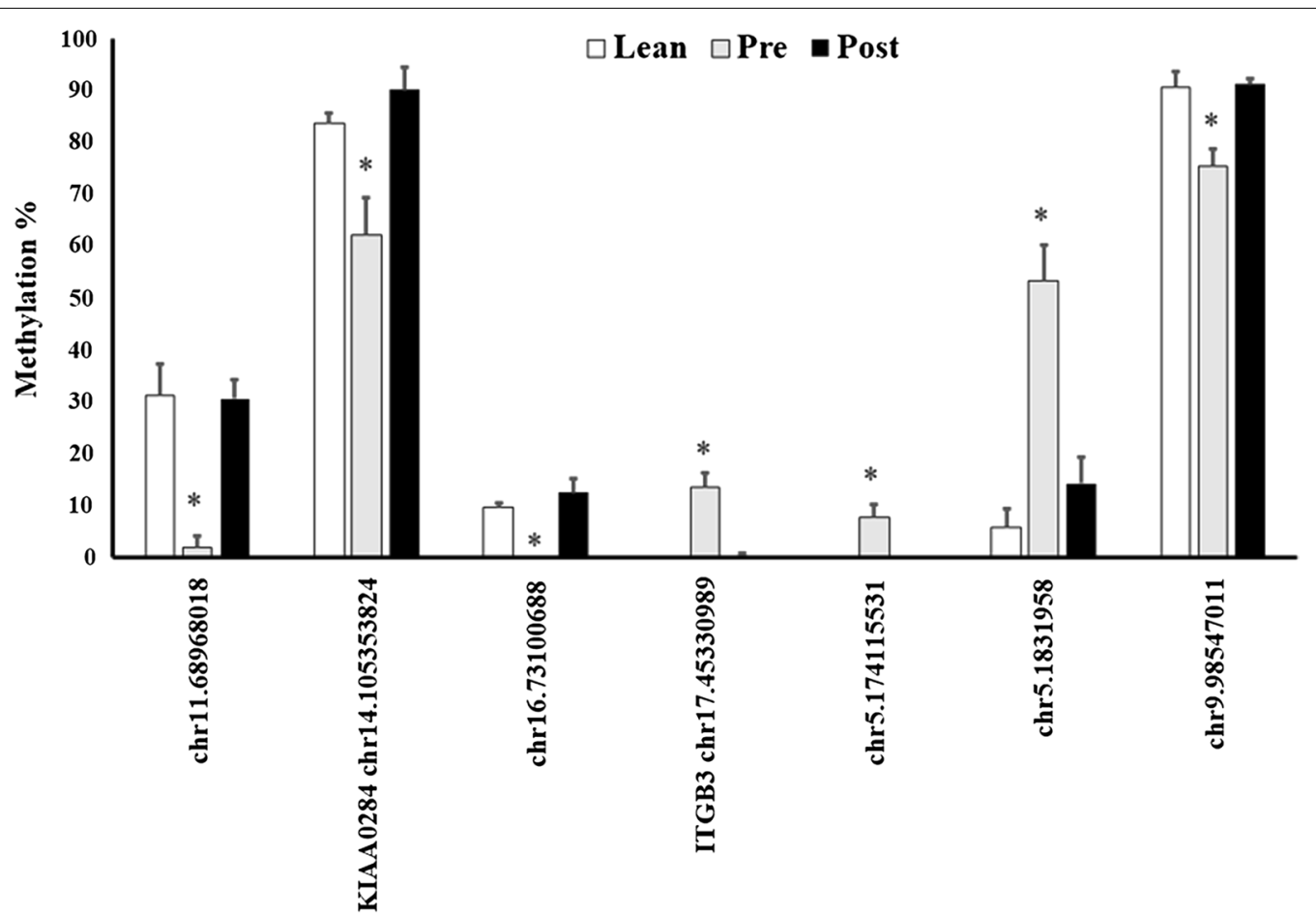

Fig. 4 Differentially methylation cytosines that post-surgery were normalized to levels similar to those observed in the lean control subjects. Data are mean \pm SEM. *Benjamini-Hochberg $q<0.05$

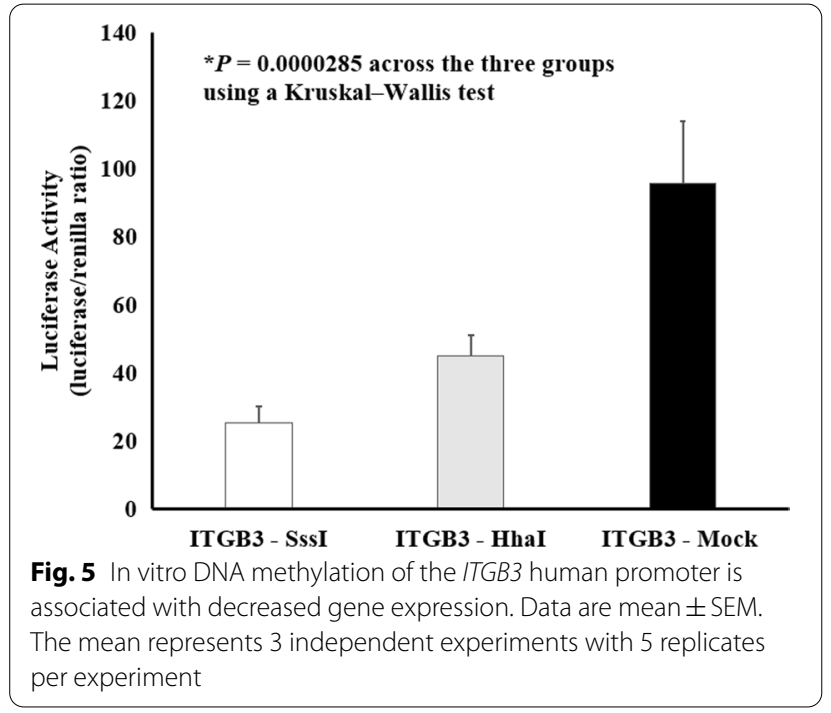

cytosines (DMCs) that were normalized post-surgery to the lean group. By including the lean control group, we were able to identify DMCs that were altered in the presurgery obese group compared to the lean.

In this study, we predicted that global methylation across the groups would be unchanged. This was in part, because Barres et al. showed that global methylation levels were similar before and after RYGB surgeryinduced weight loss, as well as being similar between the obese and nonobese women [11]. We showed that the post-surgery group had the highest global methylation at $33.31 \pm 0.02 \%$ compared to the pre-surgery at $32.97 \pm 0.02 \%$ and the lean group at $30.46 \pm 0.02 \%$. Interestingly, even though the post-surgery group had an intervention that resulted in weight loss and improvements in cholesterol, LDL, FPG and FSI, the global methylation average was significantly higher than the lean participants. In fact, the post-surgery group had a slightly higher average global methylation compared to the pre-surgery. The difference between the post- versus pre-surgery global methylation average was a change of 0.34 . Although this was a significant change, we consider the individual DMC data that is changing within these groups to be more biologically relevant than the global averages. We observed a much greater number of DMCs that were altered when comparing the pre-surgery obese to the lean control group $(n=2978)$, and the post-surgery obese to the lean control group $(n=2885)$. With the postversus pre-surgery analysis, we identified a much lower number of DMCs $(n=117)$. This finding alone suggests that the methylation changes are much more pronounced 
between the obese and lean phenotypes compared to the surgery intervention.

To our knowledge, this is one of the first studies to examine global differential DNA methylation in skeletal muscle following RYGB surgery. A study by Barres et al. measured skeletal muscle global $\mathrm{CpG}$ and nonCpG methylation in patients before and 6 months after bariatric surgery, and in lean healthy controls [11]. In that study, they showed that global methylation was unaltered by obesity or RYGB surgery. As part of that same study, they measured the promoter methylation of 14 metabolic genes and showed that 11 genes post-surgery including PPARGC1 and PDK1 were normalized to levels observed in the lean control group [11]. In the present study, we identified 7 DMCs (two DMCs associated with ITGB3 and KIAA0284) that were normalized postsurgery to lean controls. The genes that were normalized in the Barres et al. study were not in common with this study [11]. This could be due to the time of the intervention, in the Barres study the vastus lateralis was collected 6-month post-surgery while we collected the biopsy at 3 months [11]. The discrepancy in the normalized sites in the genes may be explained by the different technologies used for measuring methylation.

Our analyses identified ITGB3 as a potential gene that was altered in methylation and gene expression following weight loss induced by RYGB surgery. We showed that a differentially methylated cytosine in ITGB3 was normalized post-surgery to levels observed in the lean healthy controls, even though the mean BMI between the lean and post-surgery groups were not comparable. The DMC was located in the promoter region of ITGB3, and typically sites in regulatory regions can impact gene expression. We showed that the decreased methylation at cytosine chr17.45,330,989 in ITGB3 corresponded with an increase in gene expression post-surgery. The link between DNA methylation and gene expression changes were further established by in vitro measures using a luciferase assay. These results provide evidence for the regulatory role of promoter methylation on gene expression. We showed a stepwise decrease in luciferase expression with an increasing number of methylated sites. We observed decreased gene expression with both the methylation set by HhaI (GCGC, $n=6$ sites) and SssI (CG, $n=53$ sites). This suggests that methylation at multiple cytosines in the 1319 bp fragment of the human ITGB3 promoter (chr17:45,329,911-45,331,229) is important.

We attempted to validate ITGB3 DMC (chr17.45,330,989) using pyrosequencing (data not shown). The ITGB3 DMC (chr17.45,330,989) site of interest resides in a homopolymer T-rich region. Specifically, there are two homopolymer T-regions prior to our site of interest. These homopolymer T-regions can be a challenge for pyrosequencing experiments as discussed by Fakruddin and Chowdhury (Am J Biochem Biotechnol. 8 (1): 14-20, 2012). The results of the pyrosequencing experiments were questionable, in part, because of the dispensation of extra T's in the sequence. We speculate that this was due to the homopolymer T-regions that lie in that region. Although we do not show validation with pyrosequencing, we were able to show that ITGB3 gene expression was altered following bariatric surgery. Moreover, we showed that the ITGB3 promoter methylation in vitro significantly suppressed transcriptional activity. These additional experiments to our RRBS finding suggest that ITGB3 may be important in skeletal muscle.

In the present study, the exact mechanisms by which DNA methylation regulates the transcription of ITGB3 was not investigated. We did perform transcription factor binding analysis of the ITGB3 DMC chr17.45,330,989 to identify potential transcription factor binding motifs that may be important for influencing transcriptional regulation at this region. Paired box 5 (PAX-5), p53 and activating enhancer-binding protein 2-alpha (AP-2alphaA) overlapped with the ITGB3 site of interest. Transcription factor p53 is a critical protein involved in many aspects of human physiology [23]. A review by Kung and Murphy provided evidence that p53 is a key player in diabetes, and that activation of p53 was shown to exacerbate diabetic phenotypes [23]. The role of PAX-5 and AP2alphaA in metabolism specifically in skeletal muscle tissue is less known. Future studies are needed to determine whether DNA methylation at the ITGB3 region influences transcription factor binding and the regulation of gene expression. Moreover, additional studies are warranted to measure DNA methylation of the transcription factors themselves to determine if that impacts how they bind to the DNA, and thereby impacts expression.

Integrin subunit beta 3 (ITGB3) is part of the integrin family. Integrins are transmembrane heterodimeric glycoprotein receptors that link the extracellular matrix (ECM) to the intracellular cytoskeleton [24]. ITGB3 is most commonly expressed as a surface adhesion protein on platelets. It is an integral part of platelet aggregation by acting as a receptor for fibrinogen, von Willebrand factor, and fibronectin [25]. We performed global methylation analysis on skeletal muscle DNA, and our most significant finding was a DMC (chr17.45,330,989) in ITGB3. Recent data from numerous sources suggest that ITGB3 is important for muscle differentiation [26-28]. A recent study of the longissimus dorsi muscle of neonatal pigs showed that ITGB3 is involved in myogenic differentiation [26]. In addition, a study in C2C12 myoblasts showed that ITGB3 is upregulated during myogenic differentiation [27]. Moreover, a recent review of multiple 
transcriptome and methylome datasets in human skeletal muscle identified ITGB3 as an important gene that was upregulated and hypomethylated following acute resistance exercise [28]. In the present study, we observed an increased gene expression and decreased methylation in ITGB3 following bariatric surgery. Although exercise and bariatric surgery are different environmental exposures, it is interesting that there are similar findings with ITGB3 methylation and expression across the studies [28]. Liu et al. showed that ITGB3 is a mediator of satellite cell differentiation in regenerating muscle [27]. Skeletal muscle satellite cells play a crucial role in muscle fiber maintenance, repair and remodeling. We speculate that the ITGB3 increased gene expression may be due to the bariatric surgery having an impact on the regulation of satellite cells in human skeletal muscle. However, our study is preliminary and future studies are warranted to address the exact mechanism that ITGB3 plays on satellite/muscle cell repairing and remodeling of the skeletal muscle tissue. It could be argued that the methylation and gene expression changes observed post- versus pre-surgery in ITGB3 is coming from platelets within the skeletal muscle tissue. However, we showed no change in platelets levels following the bariatric surgery (Post-surgery: $300 \pm 34$ versus pre-surgery: $301 \pm 32$ billion/L, $P=\mathrm{NS}$ ). Moreover, platelets lack a nucleus and therefore do not contain nuclear DNA.

Although the focus of this paper was on the post-surgery DMCs that were normalized to levels observed in lean controls, there were several pathways from the presurgery obese versus lean, and the post-surgery obese versus lean analyses that warrant discussion. KEGG pathway analysis revealed an enrichment in differentially methylated cytosines within mechanosignaling and metabolic pathways for both the pre-surgery versus lean and postsurgery versus lean. These common pathways included actin cytoskeleton, focal adhesion, insulin signaling and adipocytokine signaling. Previous work from our laboratory [29-34] and others [35, 36] showed that the extracellular matrix (ECM) and the structure and function of muscle itself may be involved in the pathogenesis of insulin resistance, which is an underlying feature of obesity and type 2 diabetes. In the present study, the pre-surgery obese and post-surgery obese patients were insulin resistant compared to the lean participants, as determined by the euglycemic hyperinsulinemic clamp. Our overarching hypothesis for insulin resistant skeletal muscle is that mechanosensing and signaling of contractile forces from the ECM, to the cytoskeleton, and finally, the nucleus, is altered, leading to changes in the expression of metabolic and nuclear encoded mitochondrial genes [34]. This present study provides additional evidence to our hypothesis by identifying methylation changes in genes coding for pathways related to the ECM, cytoskeletal and metabolic genes in the pre-surgery obese and post-surgery obese compared to the lean controls.

In summary, our findings showed that there is a greater number of DNA methylation changes in skeletal muscle in the pre-surgery obese versus lean and post-surgery obese versus lean analyses, compared to the post-surgery versus pre-surgery. We observed seven DMCs that were normalized to the levels observed in lean, healthy controls. Only one of the seven DMCs was in a regulatory promoter region; therefore, we focused our downstream analyses on the ITGB3 gene. We observed a decreased methylation post-surgery that corresponded with an increase in ITGB3 gene expression. We also showed that ITGB3 promoter methylation in vitro significantly suppressed transcriptional activity. We identified several transcription factor regulatory regions within the differentially methylated ITGB3 region. However, further work is required to understand the exact mechanisms of DNA methylation on the transcriptional regulation of ITGB3.

There are limitations to this study that warrant a discussion. First, one major limitation was the small number of participants studied. Second, the study participants were exclusively female. Third, the limited size of the muscle biopsies meant we were unable to perform the quantitative real-time PCR experiment in the lean participants. Finally, we were unable to validate the ITGB3 methylation finding due to the homopolymer T-rich regions. Despite these limitations, we identified a number of genes that were differentially methylated in the pre-surgery obese versus lean, and post-surgery obese versus lean analyses to support our overarching mechanosignaling hypothesis in insulin resistant muscle. Moreover, we identified a potential gene, ITGB3 that may play an important role in muscle following bariatric surgery. Additional studies for the future include studying a larger number of participants (both male and female participants) and include multiple time points for biopsy collection. Moreover, additional studies are warranted to address the exact mechanism of action of ITGB3 in skeletal muscle metabolism.

\section{Methods \\ Participants}

Seven insulin-resistant obese $\left(B M I>40 \mathrm{~kg} / \mathrm{m}^{2}\right)$ female subjects $(45.1 \pm 3.6$ years) were studied before and three months after RYGB. The metabolic data for these subjects were included in a previous publication [5]. Studies were approved by the Institutional Review Boards at the Mayo Clinic in Arizona and Arizona State University and were performed in the Clinical Studies Infusion Unit (CSIU) at Mayo Clinic in Arizona. After giving informed written consent, each volunteer underwent a medical 
history, physical examination, screening laboratory tests, a 75-g oral glucose tolerance test. On a different day, participants reported fasting to the CSIU to undergo an $80 \mathrm{mU} / \mathrm{m}^{2}$ euglycemic-hyperinsulinemic clamp with muscle biopsies collected before insulin infusion, as previously described $[5,37]$. Three months post-RYGB the participants returned to the CSIU and a repeat euglycemic-hyperinsulinemic clamp with muscle biopsy was performed. The Roux-en-Y gastric bypass was performed at the Bariatric Surgery Program at the Mayo Clinic in Arizona [5]. Details of the program and the surgical procedures were described in our previous publication [5].

\section{Substrate and hormone determinations}

The Biospecimens Accessioning and Processing Core at the Mayo Clinic in Arizona performed the screening laboratory tests and metabolic panel. Plasma glucose levels were measured by the YSI 2300 STAT plus (YSI INC., Yellow Sprigs, $\mathrm{OH}$ ) in the CSIU. Serum insulin was measured at the Immunochemical Core Laboratory (ICL) at the Mayo Clinic in Rochester. The d2-glucose enrichment data from the euglycemic hyperinsulinemic clamp were measured at the Center for Clinical and Translational Science (CCaTS) Metabolomics Core at the Mayo Clinic in Rochester.

\section{Muscle biopsy processing}

Homogenization of the muscle biopsy (25 mg) was performed in 1X PBS with the Bullet Blender (Integrated Scientific Solutions, San Diego, CA). DNA was isolated using QIAamp DNA mini kit, as per the manufacturer's instructions (Qiagen, Valencia, CA). DNA quantity and quality was assessed using agarose gel electrophoresis and spectrophotometer A260/A280 values were determined using the NanoVue (GE Healthcare, UK).

\section{Reduced representation bisulfite sequencing (RRBS) next generation sequencing}

RRBS was performed on DNA at the Mayo Clinic Genotyping Shared Resource facility as previously described $[38,39]$. Sequencing data were analyzed using a streamlined analysis and annotation pipeline for reduced representation bisulfite sequencing, SAAP-RRBS [39]. The methylation dataset supporting the conclusions of this article are available in the Gene Expression Omnibus repository, GSE164305 (http://www.ncbi.nlm.nih.gov/ geo/).

\section{Differentially methylated cytosines (DMC) analysis}

To determine differences in methylation sites between groups, the aligned ( $\mathrm{Hg} 19)$ data were imported into the free open source R package, MethylSig [40]. A minimum of five reads and the recovery of the site in all participants from each group were required for the inclusion of a cytosine in subsequent analyses. The mean methylation differences (\%) were determined as previously described, with a Benjamini-Hochberg multiple testing correction applied to the data. Regional annotations for each DMC were imported from the University of California, Santa Cruz (UCSC) Genome Browser's RefSeq Genes and CpG Island tracks. Priority was given to annotating the site as a promoter or untranslated region if available in another transcript of the gene or in a different gene. The Database for Annotation, Visualization and Integrated Discovery (DAVID) pathway analysis was performed on the DMC data $[41,42]$.

\section{ITGB3 quantitative RT-PCR}

Skeletal muscle gene expression for ITGB3 was detected using quantitative real-time PCR on the ABI PRISM 7900HT sequence detection system (Life Technologies, Carlsbad, CA). TaqMan Universal Fast PCR master mix reagents and the Assay-On-Demand gene expression primer pair and probes (Life Technologies, Carlsbad, CA) were added to $50 \mathrm{ng}$ cDNA, which was synthesized using the ABI High Capacity cDNA Reverse Transcription Kit, as per manufacturer's instructions.

The quantity of ITGB3 (Hs01001469_m1) in each sample was normalized to 18S (Hs99999901_s1) using the comparative (2- $\Delta \mathrm{CT})$ method [43].

\section{ITGB3 luciferase assay}

A 1319 bp fragment of the human ITGB3 promoter (chr17:45,329,911-45,331,229) was cloned into a CpG free luciferase reporter vector (pCpGL-basic). Briefly, the ITGB3 promoter sequence was identified by searching the gene sequence in the genome web browser ensembl (www.ensembl.org). The transcription start site (TSS) was identified after downloading the gene sequence from the database. We selected the first $1500 \mathrm{bp}$ upstream of the TSS and entered it into the UCSC BLAT (https:// genome.ucsc.edu/cgi-bin/hgBlat). We did this to confirm whether the promoter sequence given by ensembl matched the sequence that is annotated in UCSC's genome browser. The best primer design for the $1500 \mathrm{bp}$ upstream sequence was selected using integrated DNA technologies (IDT) primer design tool (https://www. idtdna.com). The promoter sequence was amplified using the following primers: ITGB3-F-BamHI: ACTAGTGGA TCCATACTTGCTGAGGCCAGTGC and ITGB3-RNcoI: CTTAGTCCATGGAGCCTCACTCACCTCCTA CG. The restriction enzymes BamHI and NcoI were used to digest the PCR product, along with the CpG free

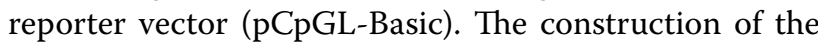
plasmid was completed by ligating the digestion products using T4 DNA ligase. Propagation of the constructed 
plasmid was accomplished through transformation of chemically competent GT115 E. coli cells. The sequence of the constructs was validated via DNA sequencing.

The ITGB3 construct was either mock methylated or methylated using $1600 \mu \mathrm{M} \mathrm{S}$-adenosylmethionine (SAM) and two different DNA methyltransferases, SssI and HhaI (New England Biolabs, Frankfurt, Germany). Mouse muscle cell lines $\mathrm{C} 2 \mathrm{C} 12$ were cultured in DMEM, supplemented with $10 \%$ serum and $1 \%$ of an antibiotic/antimycotic mixture. Cells were co-transfected with $100 \mathrm{ng}$ of pCpGL-basic with the ITGB3 promoter insert or without (control) and 2 ng of pRL renilla luciferase control reporter vector using the Lipofectamine 3000 transfection reagent (Invitrogen, Carlsbad, CA). Firefly luciferase activity was measured and normalized against the renilla luciferase activity using the Dual Luciferase Reporter Assay System (Promega, Madison, WI).

\section{ITGB3 predicted transcription factor binding analysis}

Transcription factor binding sites analysis was performed using PROMO version $3.0[44,45]$. The ITGB3 sequence on chromosome 17: 45,330,979-45,330,999 encompasses the significant DMC 45,330,989, and that region was assessed for transcription factor binding sites. The sequence was analyzed with a $5 \%$ maximum matrix dissimilarity rate using TRANSFAC version 8.3 database.

\section{Statistical analysis}

Data were presented as a mean \pm standard error of the mean (SEM). Statistical comparisons of the pre- versus post-surgery characteristic data were performed using a paired Student's $t$-test. Statistical comparisons of the pre-surgery versus lean and post-surgery versus lean characteristic data was performed using an unpaired Student's $t$-test. Global methylation data were presented as the means \pm SEM. Significance was assessed using a nonparametric Wilcoxon signed-rank test for the paired pre- versus post-surgery global methylation analysis. For the unpaired lean versus pre-surgery and the unpaired lean versus post-surgery global methylation analysis, we performed the nonparametric Mann-Whitney $U$ test. MethylSig was used for determining differential methylated cytosines (DMCs) in the reduced representation bisulfite sequencing (RRBS) data. MethylSig uses a beta binomial model to test for significant differences between groups of samples. A comparison of the DNA methylation between the groups at each site was based on a likelihood ratio test (nominal $P$ Value), and a Benjamini-Hochberg $(q<0.05)$ multiple testing correction was applied in order to reduce false positives. For the quantitative RT-PCR analyses, the quantity of ITGB3 was normalized to $18 \mathrm{~S}$ using the comparative $(2-\Delta \Delta \mathrm{CT})$ method [43] and significance was assessed using a paired
Student's $t$-test. The luciferase data as measured by the luciferase/renilla ratio had a non-normal distribution; therefore, significance across the three groups was determined using the nonparametric Kruskal-Wallis test.

\section{Abbreviations}

DMC: Differentially methylated cytosine; RYGB: Roux-en-Y gastric bypass; RRBS: Reduced representation bisulfite sequencing; ITGB3: Integrin beta 3; BMI: Body mass index; LDL: Low-density lipoprotein; FPG: Fasting plasma glucose; FSI: Fasting serum insulin; HDL: High-density lipoprotein; HbA1c: Hemoglobin A1c; M-value: Insulin-stimulated glucose disposal.

\section{Supplementary Information}

The online version contains supplementary material available at https://doi. org/10.1186/s13148-021-01086-6.

Additional file 1. Gene ontology analysis on the genes with significantly decreased DMC in the post-surgery data versus pre-surgery.

Additional file 2. Gene ontology analysis on the genes with significantly increased DMC in the post-surgery data versus pre-surgery.

Additional file 3. Differentially methylated cytosines $(n=2978)$ significantly altered in methylation pre-surgery obese versus lean (Benjamini Hochberg $q<0.05)$.

Additional file 4. KEGG pathway analysis on the genes with significantly decreased DMC in the pre-surgery obese versus lean.

Additional file 5. KEGG pathway analysis on the genes with significantly increased DMC in the pre-surgery obese versus lean.

Additional file 6. Differentially methylated cytosines $(n=2885)$ significantly altered in methylation post-surgery obese versus lean (Benjamini Hochberg $q<0.05)$.

Additional file 7. KEGG pathway analysis on the genes with significantly decreased DMC in the post-surgery obese versus lean.

Additional file 8. KEGG pathway analysis on the genes with significantly increased DMC in the post-surgery obese versus lean.

\section{Acknowledgements}

We thank the volunteers of the study and are grateful for their participation and cooperation. We thank the Clinical Studies Infusion Unit nurses and research staff for their excellent care of the participants. We thank the Mayo Clinic Genotyping Shared Resource facility for the RRBS next generation methylation analysis.

\section{Authors' contributions}

DKC conceived the experiments. LAG, SED and DKC carried out the experiments. BC, RLC, LJM and DKC performed the analysis of the data. TRB, EDF and LRR performed the euglycemic hyperinsulinemic clamps with muscle biopsies. JAM II performed the bariatric surgery. DKC wrote the article. LAG, $B C, S E D, R L C, T R B, E D F, J A M I I, L J M$ and LRR read the manuscript and provided comments. DKC is the guarantor of this work and, as such, had full access to all the data in the study and takes responsibility for the integrity of the data and the accuracy of the data analysis. All authors read and approved the final manuscript.

\section{Funding}

This study was supported by the National Institutes of Health Grants R01DK094013 (DKC) and a Mayo/ASU seed grant (DKC, LRR).

\section{Availability of data and materials}

The methylation dataset supporting the conclusions of this article are available in the Gene Expression Omnibus repository, GSE164305 (http://www. ncbi.nlm.nih.gov/geo/). 


\section{Declarations}

\section{Ethics approval and consent to participate}

All subjects gave informed written consent to participate in the study, which was approved by the Institutional Review Boards of the Mayo Clinic in Arizona and Arizona State University (IRB 12-009688 and IRB 11-007028).

\section{Consent for publication}

Not applicable.

\section{Competing interests}

The authors declare that they have no competing interests.

\section{Author details}

1 Department of Medicine, Division of Endocrinology, The University of Arizona College of Medicine, 1501 N. Campbell Ave, PO Box 245035, Tucson, AZ 85724-5035, USA. ${ }^{2}$ Phoenix Epidemiology and Clinical Research Branch, National Institute of Diabetes and Digestive and Kidney Diseases, National Institutes of Health, Phoenix, AZ, USA. ${ }^{3}$ Department of Endocrinology, Metabolism and Diabetes, Mayo Clinic Arizona, Scottsdale, AZ, USA. ${ }^{4}$ Mayo Clinic Hospital, Phoenix, AZ, USA. ${ }^{5}$ Department of Physiology, The University of Arizona College of Medicine, Tucson, AZ, USA.

Received: 8 January 2021 Accepted: 21 April 2021

Published online: 01 May 2021

\section{References}

1. Bluher M. Obesity: global epidemiology and pathsogenesis. Nat Rev Endocrinol. 2019;15(5):288-98.

2. Hales CM, Carroll MD, Fryar CD, Ogden CL. Prevalence of obesity and severe obesity among adults: United States, 2017-2018. NCHS Data Brief. 2020;360:1-8.

3. Hruby A, Hu FB. The epidemiology of obesity: a big picture. Pharmacoeconomics. 2015;33(7):673-89.

4. Czech MP. Insulin action and resistance in obesity and type 2 diabetes. Nat Med. 2017;23(7):804-14.

5. Campbell LE, Langlais PR, Day SE, Coletta RL, Benjamin TR, De Filippis EA et al. Identification of novel changes in human skeletal muscle proteome after Roux-en-Y gastric bypass surgery. Diabetes. 2016;65(9):2724-31.

6. Maciejewski ML, Arterburn DE, Van Scoyoc L, Smith VA, Yancy WS Jr, Weidenbacher $\mathrm{HJ}$, et al. Bariatric surgery and long-term durability of weight loss. JAMA Surg. 2016;151(11):1046-55

7. le Roux CW, Heneghan HM. Bariatric surgery for obesity. Med Clin North Am. 2018;102(1):165-82.

8. Mingrone G, Castagneto-Gissey L. Mechanisms of early improvement/ resolution of type 2 diabetes after bariatric surgery. Diabetes Metab. 2009;35(6 Pt 2):518-23.

9. Mulla CM, Middelbeek RJW, Patti ME. Mechanisms of weight loss and improved metabolism following bariatric surgery. Ann NY Acad Sci. 2018;1411(1):53-64.

10. Sjostrom L. Review of the key results from the Swedish Obese Subjects (SOS) trial-a prospective controlled intervention study of bariatric surgery. J Intern Med. 2013;273(3):219-34.

11. Barres R, Kirchner H, Rasmussen M, Yan J, Kantor FR, Krook A, et al. Weight loss after gastric bypass surgery in human obesity remodels promoter methylation. Cell Rep. 2013;3(4):1020-7.

12. Guidone C, Manco M, Valera-Mora E, laconelli A, Gniuli D, Mari A, et al. Mechanisms of recovery from type 2 diabetes after malabsorptive bariatric surgery. Diabetes. 2006;55(7):2025-31.

13. Ferrannini E, Mingrone G. Impact of different bariatric surgical procedures on insulin action and beta-cell function in type 2 diabetes. Diabetes Care. 2009;32(3):514-20.

14. Buchwald H, Estok R, Fahrbach $K$, Banel D, Jensen MD, Pories WJ, et al. Weight and type 2 diabetes after bariatric surgery: systematic review and meta-analysis. Am J Med. 2009;122(3):248-56.

15. Chang L, Chiang SH, Saltiel AR. Insulin signaling and the regulation of glucose transport. Mol Med. 2004;10(7-12):65-71.
16. Haeusler RA, McGraw TE, Accili D. Biochemical and cellular properties of insulin receptor signalling. Nat Rev Mol Cell Biol. 2018;19(1):31-44.

17. Saltiel AR, Kahn CR. Insulin signalling and the regulation of glucose and lipid metabolism. Nature. 2001;414(6865):799-806.

18. White MF. Insulin signaling in health and disease. Science. 2003;302(5651):1710-1.

19. White MF, Kahn CR. The insulin signaling system. J Biol Chem. 1994;269(1):1-4.

20. Day SE, Garcia LA, Coletta RL, Campbell LE, Benjamin TR, De Filippis EA, et al. Alterations of sorbin and SH3 domain containing 3 (SORBS3) in human skeletal muscle following Roux-en-Y gastric bypass surgery. Clin Epigenetics. 2017;9:96.

21. Gastaldi G, Russell A, Golay A, Giacobino JP, Habicht F, Barthassat V, et al. Upregulation of peroxisome proliferator-activated receptor gamma coactivator gene (PGC1A) during weight loss is related to insulin sensitivity but not to energy expenditure. Diabetologia. 2007;50(11):2348-55.

22. Albers PH, Bojsen-Moller KN, Dirksen C, Serup AK, Kristensen DE, Frystyk $J$, et al. Enhanced insulin signaling in human skeletal muscle and adipose tissue following gastric bypass surgery. Am J Physiol Regul Integr Comp Physiol. 2015:309(5):R510-24.

23. Kung CP, Murphy ME. The role of the p53 tumor suppressor in metabolism and diabetes. J Endocrinol. 2016;231(2):R61-75.

24. Danen EHJ. Integrins and development. Landes Bioscience/Eurekah.com; 2006.

25. Joo SJ. Mechanisms of platelet activation and integrin alphallbeta3. Korean Circ J. 2012;42(5):295-301.

26. Zhang X, Wang L, Qiu K, Xu D, Yin J. Dynamic membrane proteome of adipogenic and myogenic precursors in skeletal muscle highlights EPHA2 may promote myogenic differentiation through ERK signaling. FASEB J. 2019;33(4):5495-509.

27. Liu H, Niu A, Chen SE, Li YP. Beta3-integrin mediates satellite cell differentiation in regenerating mouse muscle. FASEB J. 2011;25(6):1914-21.

28. Turner DC, Seaborne RA, Sharples AP. Comparative transcriptome and methylome analysis in human skeletal muscle anabolism, hypertrophy and epigenetic memory. Sci Rep. 2019;9(1):4251.

29. Berria R, Wang L, Richardson DK, Finlayson J, Belfort R, Pratipanawatr T, et al. Increased collagen content in insulin-resistant skeletal muscle. Am J Physiol Endocrinol Metab. 2006;290(3):E560-5.

30. Hwang $H$, Bowen BP, Lefort N, Flynn CR, De Filippis EA, Roberts C, et al. Proteomics analysis of human skeletal muscle reveals novel abnormalities in obesity and type 2 diabetes. Diabetes. 2010;59(1):33-42.

31. Lefort N, Yi Z, Bowen B, Glancy B, De Filippis EA, Mapes R, et al. Proteome profile of functional mitochondria from human skeletal muscle using one-dimensional gel electrophoresis and HPLC-ESI-MS/MS. J Proteomics. 2009;72(6):1046-60.

32. Patti ME, Butte AJ, Crunkhorn S, Cusi K, Berria R, Kashyap S, et al. Coordinated reduction of genes of oxidative metabolism in humans with insulin resistance and diabetes: potential role of PGC1 and NRF1. Proc Natl Acad Sci USA. 2003;100(14):8466-71.

33. Richardson DK, Kashyap S, Bajaj M, Cusi K, Mandarino SJ, Finlayson J, et al. Lipid infusion decreases the expression of nuclear encoded mitochondrial genes and increases the expression of extracellular matrix genes in human skeletal muscle. J Biol Chem. 2005:280(11):10290-7.

34. Coletta DK, Mandarino LJ. Mitochondrial dysfunction and insulin resistance from the outside in: extracellular matrix, the cytoskeleton, and mitochondria. Am J Physiol Endocrinol Metab. 2011;301(5):749-55.

35. Williams AS, Kang L, Wasserman DH. The extracellular matrix and insulin resistance. Trends Endocrinol Metab. 2015;26(7):357-66.

36. Martinez-Huenchullan S, McLennan SV, Verhoeven A, Twigg SM, Tam CS The emerging role of skeletal muscle extracellular matrix remodelling in obesity and exercise. Obes Rev. 2017;18(7):776-90.

37. DeFronzo RA, Tobin JD, Andres R. Glucose clamp technique: method for quantifying insulin secretion and resistance. Am J Physiol. 1979:237(3):E214-23.

38. Day SE, Coletta RL, Kim JY, Campbell LE, Benjamin TR, Roust LR, et al. Nextgeneration sequencing methylation profiling of subjects with obesity identifies novel gene changes. Clin Epigenetics. 2016;8:77.

39. Sun Z, Baheti S, Middha S, Kanwar R, Zhang Y, Li X, et al. SAAP-RRBS: streamlined analysis and annotation pipeline for reduced representation bisulfite sequencing. Bioinformatics. 2012;28(16):2180-1. 
40. Park Y, Figueroa ME, Rozek LS, Sartor MA. MethylSig: a whole genome DNA methylation analysis pipeline. Bioinformatics. 2014;30(17):2414-22.

41. Huang DW, Sherman BT, Tan Q, Collins JR, Alvord WG, Roayaei J, et al. The DAVID Gene Functional Classification Tool: a novel biological modulecentric algorithm to functionally analyze large gene lists. Genome Biol. 2007;8(9):R183.

42. Huang DW, Sherman BT, Tan Q, Kir J, Liu D, Bryant D, et al. DAVID Bioinformatics Resources: expanded annotation database and novel algorithms to better extract biology from large gene lists. Nucleic Acids Res. 2007;35:W169-75.

43. Livak KJ, Schmittgen TD. Analysis of relative gene expression data using real-time quantitative PCR and the 2(-Delta Delta C(T)) method. Methods. 2001;25(4):402-8
44. Farre D, Roset R, Huerta M, Adsuara JE, Rosello L, Alba MM, et al. Identification of patterns in biological sequences at the ALGGEN server: PROMO and MALGEN. Nucleic Acids Res. 2003;31(13):3651-3.

45. Messeguer X, Escudero R, Farre D, Nunez O, Martinez J, Alba MM. PROMO: detection of known transcription regulatory elements using speciestailored searches. Bioinformatics. 2002;18(2):333-4.

\section{Publisher's Note}

Springer Nature remains neutral with regard to jurisdictional claims in published maps and institutional affiliations.
Ready to submit your research? Choose BMC and benefit from:

- fast, convenient online submission

- thorough peer review by experienced researchers in your field

- rapid publication on acceptance

- support for research data, including large and complex data types

- gold Open Access which fosters wider collaboration and increased citations

- maximum visibility for your research: over 100M website views per year

At BMC, research is always in progress.

Learn more biomedcentral.com/submissions 